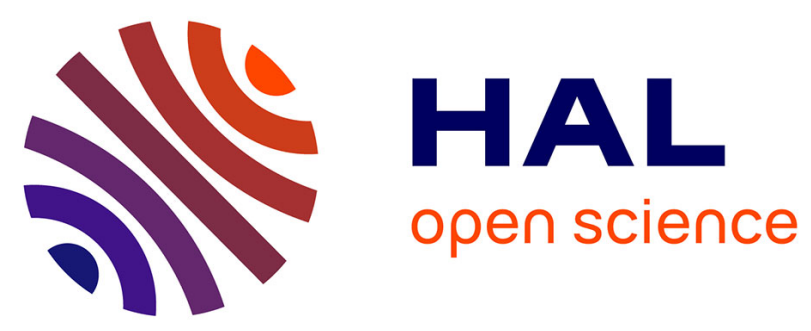

\title{
Local existence and uniqueness of the mild solution to the 1D Vlasov-Poisson system with an initial condition of bounded variation
}

\author{
Simon Labrunie, Sandrine Marchal, Jean Rodolphe Roche
}

\section{- To cite this version:}

Simon Labrunie, Sandrine Marchal, Jean Rodolphe Roche. Local existence and uniqueness of the mild solution to the 1D Vlasov-Poisson system with an initial condition of bounded variation. Mathematical Methods in the Applied Sciences, 2010, 33 (17), pp.2132-2142. 10.1002/mma.1328 . hal-00397497v2

\section{HAL Id: hal-00397497 \\ https://hal.science/hal-00397497v2}

Submitted on 6 Apr 2010

HAL is a multi-disciplinary open access archive for the deposit and dissemination of scientific research documents, whether they are published or not. The documents may come from teaching and research institutions in France or abroad, or from public or private research centers.
L'archive ouverte pluridisciplinaire HAL, est destinée au dépôt et à la diffusion de documents scientifiques de niveau recherche, publiés ou non, émanant des établissements d'enseignement et de recherche français ou étrangers, des laboratoires publics ou privés. 


\title{
Local existence and uniqueness of the mild solution to the 1D Vlasov-Poisson system with an initial condition of bounded variation
}

\author{
Simon Labrunie*, Sandrine Marchal, Jean-Rodolphe Roche
}

We propose a result of local existence and uniqueness of a mild solution to the one-dimensional Vlasov-Poisson system. We establish the result for an initial condition lying in the space $W^{1,1}\left(\mathbb{R}^{2}\right)$, then we extend it to initial conditions lying in the space $B V\left(\mathbb{R}^{2}\right)$, without any assumption of continuity, boundedness or compact support. Copyright (C) 2010 John Wiley \& Sons, Ltd.

Keywords: Vlasov-Poisson system, bounded variation functions, Banach contraction mapping principle

\section{Introduction}

\subsection{Position of the problem}

In this paper we study the one-dimensional Vlasov-Poisson system:

$$
\begin{array}{ll}
\forall(t, x, v) \in[0, T] \times \mathbb{R}^{2}, & \frac{\partial f}{\partial t}(t, x, v)+v \frac{\partial f}{\partial x}(t, x, v)+E(t, x) \frac{\partial f}{\partial v}(t, x, v)=0, \\
\forall(t, x) \in[0, T] \times \mathbb{R}, & \frac{\partial E}{\partial x}(t, x)=\int_{\mathbb{R}} f(t, x, v) d v-n_{\mathrm{b}}(t, x), \\
\forall(x, v) \in \mathbb{R}^{2}, & f(0, x, v)=f_{0}(x, v) .
\end{array}
$$

This system models the behaviour of a gas of charged particles in an electrostatic field, created by the particles and by a so-called neutralising background, whose density $n_{\mathrm{b}}$ is assumed to be given. Collisions between particles are neglected. In [7], Cooper and Klimas show the existence and uniqueness of a global mild solution to this system, i.e. a solution defined by characteristics, for a continuous and bounded initial condition $f_{0}$ which has its first two moments in $v$ uniformly bounded in $x$, and a constant background density $n_{\mathrm{b}}$. This was extended by Bostan [5] to the initial-boundary value problem, with slightly more general hypotheses on the initial and boundary conditions, namely, that they are bounded but not necessarily continuous, and have one moment in $v$ uniformly bounded in $x$. In [12], Guo showed that there exists a unique local weak solution to (1-3) in the space $L^{\infty}\left([0, T], B V\left(\mathbb{R}^{2}\right)\right)$ for initial and boundary conditions with compact support and in the space $L^{\infty}\left(\mathbb{R}^{2}\right) \cap B V\left(\mathbb{R}^{2}\right)$. (The latter two works do not consider a neutralising background, but can be easily extended to this situation.)

In higher dimensions, the known existence and uniqueness results usually rest upon restrictive assumptions. To get strong or mild solutions, the standard hypotheses on $f_{0}$ are either explicit decay bounds in $v$ (of the form $C(1+|v|)^{-\alpha}$ ) for $f_{0}$ and its first derivatives [13], or local boundedness and Lipschitz-style conditions together with a finite energy [17], or even a bounded (at least in $v$ ) support $[2,19]$. As for weak or renormalised solutions, their existence is generally proven under assumptions including $f_{0} \in L^{1}\left(\mathbb{R}^{2 d}\right) \cap L^{p}\left(\mathbb{R}^{2 d}\right)$, with $p$ large enough, and a certain number of global moments in $v$ finite (at least $\iint|v|^{2} f_{0} d x d v$, corresponding to kinetic energy); see Horst-Hunze [14], DiPerna-Lions [8], Lions-Perthame [15]. Uniqueness results generally assume, once more, a compact support [18], or the boundedness of the spatial density [16], sometimes complemented by 
Lipschitz-style conditions [15]. Let us finally mention the existence and uniqueness result of compactly supported solutions in $L^{1}\left(\mathbb{R}^{6}\right) \cap L^{\infty}\left(\mathbb{R}^{6}\right)$ by Zhidkov [21].

In this article we extend the results of Guo to the initial value problem with intial data in the space $B V\left(\mathbb{R}^{2}\right)$, thus not necessarily compactly supported, bounded, or continuous. As far as Lebesgue regularity is concerned, this implies $f_{0} \in L^{1}\left(\mathbb{R}^{2}\right) \cap L^{2}\left(\mathbb{R}^{2}\right)$. Our proof is based on the contraction mapping principle of Banach, and consists of two steps: first we establish the local existence and uniqueness of a mild solution for an initial data $f_{0}$ in $W^{1,1}\left(\mathbb{R}^{2}\right)$, then we extend the result to $f_{0} \in B V\left(\mathbb{R}^{2}\right)$.

\subsection{Notations and main results}

We introduce the following notations (see [12]). Given $T>0$, we denote

$$
U_{T}=(0, T) \times \mathbb{R} \text { and } V_{T}=(0, T) \times \mathbb{R}^{2} .
$$

For $s \in[0, T]$, we denote $\Pi_{s}=\{s\} \times \mathbb{R}^{2}$ the slice $t=s$ of $\overline{V_{T}}$. Then we introduce the following functional spaces:

$$
L(T)=L^{\infty}\left(0, T ; W^{1,1}\left(\mathbb{R}^{2}\right)\right), \quad X(T)=L^{\infty}\left(0, T ; W^{1, \infty}(\mathbb{R})\right) .
$$

The space $L(T)$ will be that of the solutions $f$ to the Vlasov equation with initial data $f_{0} \in W^{1,1}\left(\mathbb{R}^{2}\right)$; we equip it with its natural norm. As for $X(T)$, it is a space of electrostatic fields $E$ for which the characteristic curves are globally well defined and Lipschitz-continuous in all their variables [5]. This can be shown by adapting the proof of the Cauchy-Lipschitz theorem: the only difference is that we integrate $L^{\infty}$ functions instead of $C^{0}$ functions and so we get continuous solutions differentiable almost everywhere in the time variable and with bounded derivative. We equip it with the following norm:

$$
\forall E \in X(T), \quad\|E\|_{X(T)}=\max \left(\|E\|_{L^{\infty}\left(U_{T}\right)},\left\|\partial_{X} E\right\|_{L^{\infty}\left(U_{T}\right)}\right) .
$$

Morover, for any $E \in X(T)$, we set

$$
C(E)=\max \left(\left\|\partial_{x} E\right\|_{L^{\infty}\left(U_{T}\right)}, 1\right)
$$

and we denote by $Y_{E}$ the Vlasov differential operator:

$$
Y_{E}=\frac{\partial}{\partial t}+v \frac{\partial}{\partial t}+E(t, x) \frac{\partial}{\partial v}
$$

We recall the definition of the total variation of a function $f \in L^{1}\left(\mathbb{R}^{2}\right)$ (see for example [9, p. 39]):

$$
\forall f \in L^{1}\left(\mathbb{R}^{2}\right), \quad T V[f]=T V_{x}[f]+T V_{v}[f],
$$

where:

$$
\begin{aligned}
& T V_{x}[f]=\limsup _{\epsilon \longrightarrow 0} \int_{-\infty}^{+\infty} \int_{-\infty}^{+\infty}\left|\frac{f(x+\epsilon, v)-f(x, v)}{\epsilon}\right| d x d v \\
& T V_{v}[f]=\limsup _{\epsilon \longrightarrow 0} \int_{-\infty}^{+\infty} \int_{-\infty}^{+\infty}\left|\frac{f(x, v+\epsilon)-f(x, v)}{\epsilon}\right| d x d v
\end{aligned}
$$

The space of functions of bounded variation is defined as:

$$
B V\left(\mathbb{R}^{2}\right)=\left\{f \in L^{1}\left(\mathbb{R}^{2}\right): T V[f]<+\infty\right\}
$$

and equipped with the norm $\|f\|_{B V\left(\mathbb{R}^{2}\right)}=\|f\|_{L^{1}\left(\mathbb{R}^{2}\right)}+T V[f]$.

Finally, we denote by $L^{b v}(T)$ the space $L^{\infty}\left(0, T ; B V\left(\mathbb{R}^{2}\right)\right)$ equipped with its natural norm. We shall establish the following two theorems:

Theorem 1 (Local existence and uniqueness in $W^{1,1}$ ) Let $f_{0}$ and $n_{\mathrm{b}}$ be non-negative given functions in $W^{1,1}\left(\mathbb{R}^{2}\right)$ and $L_{\text {loc }}^{\infty}\left(\mathbb{R}^{+} ; L^{1}(\mathbb{R}) \cap L^{\infty}(\mathbb{R})\right)$, respectively. For any $(R, T)$ such that:

$$
\begin{aligned}
R & \geq \max \left(\frac{1}{2}\left[\left\|f_{0}\right\|_{L^{1}\left(\mathbb{R}^{2}\right)}+\left\|n_{\mathrm{b}}\right\|_{L^{\infty}\left(0, T ; L^{1}(\mathbb{R})\right)}\right],\left\|n_{\mathrm{b}}\right\|_{L^{\infty}\left(U_{T}\right)},\left|f_{0}\right|_{W^{1,1}\left(\mathbb{R}^{2}\right)}, 1\right) \\
T & \in\left[0, \frac{1}{R} \ln \left(\frac{R}{\left|f_{0}\right|_{W^{1,1}\left(\mathbb{R}^{2}\right)}}\right)\right],
\end{aligned}
$$

there exists a unique mild solution $(f, E) \in L(T) \times X(T)$ to $(1-3)$. 
Moreover, we have a lower bound on the existence time $T_{\text {ex }}$ of the maximal solution to (1-3). Let $T_{*}>0$ be chosen arbitrarily to bound the various norms of $n_{\mathrm{b}}$, and set $R_{0}:=\max \left(\frac{1}{2}\left(\left\|f_{0}\right\|_{L^{1}\left(\mathbb{R}^{2}\right)}+\left\|n_{\mathrm{b}}\right\|_{L^{\infty}\left(0, T_{*} ; L^{1}(\mathbb{R})\right)}\right),\left\|n_{\mathrm{b}}\right\|_{L^{\infty}\left(U_{T_{*}}\right)}, 1\right)$. Then:

$$
\begin{aligned}
& T_{\text {ex }} \geq \min \left(T_{*}, \frac{1}{(\mathrm{e}-1)\left|f_{0}\right|_{W^{1,1}}}\right) \quad \text { if }\left|f_{0}\right|_{W^{1,1}} \geq \frac{R_{0}}{\mathrm{e}} \\
& T_{\text {ex }} \geq \min \left(T_{*}, \frac{1}{R_{0}}\left[\ln \frac{R_{0}}{\left|f_{0}\right|_{W^{1,1}}}+\frac{1}{(\mathrm{e}-1)}\right]\right) \quad \text { if } \quad\left|f_{0}\right|_{W^{1,1}} \leq \frac{R_{0}}{\mathrm{e}}
\end{aligned}
$$

If the $W^{1,1}$ norm of $f(t)$, or equivalently the $L^{\infty}$ norm of the density $n(t):=\int f(t) d v$, remains bounded for $t \in\left(0, T_{\mathrm{ex}}\right)$, then $T_{\text {ex }}=+\infty$, i.e. the solution is global. Otherwise, the following bounds hold:

$$
|f(t)|_{W^{1,1}} \geq \frac{1}{(\mathrm{e}-1)\left(T_{\mathrm{ex}}-t\right)}, \quad\|n(t)\|_{L \infty} \geq C\left|\ln \left(T_{\mathrm{ex}}-t\right)\right|
$$

for some constant $C$ and $T_{\text {ex }}-t$ small enough.

Theorem 2 (Local existence and uniqueness in $B V$ ) Assume now that $f_{0} \in B V\left(\mathbb{R}^{2}\right)$, while keeping all other hypotheses of Theorem 1. If $(R, T)$ are such that:

$$
\begin{aligned}
& R \geq \max \left(\frac{1}{2}\left[\left\|f_{0}\right\|_{L^{1}\left(\mathbb{R}^{2}\right)}+\left\|n_{\mathrm{b}}\right\|_{L^{\infty}\left(0, T ; L^{1}(\mathbb{R})\right)}\right],\left\|n_{\mathrm{b}}\right\|_{L^{\infty}\left(U_{T}\right)}, T V\left[f_{0}\right], 1\right) \\
& T \in\left[0, \frac{1}{R} \ln \left(\frac{R}{T V\left[f_{0}\right]}\right)\right]
\end{aligned}
$$

there exists a unique mild solution $(f, E) \in L^{\text {bv }}(T) \times X(T)$ to (1-3). The bounds (10), (11), (12) hold, with the semi-norms $\left|f_{0}\right|_{W^{1,1},}|f(t)|_{W^{1,1}}$ replaced with total variations $T V\left[f_{0}\right], \operatorname{TV}[f(t)]$

The proof is organised as follows. In $\S 2$, we recall the definitions of weak and mild solutions to the linear Vlasov equation (i.e. (1) and (3) with $E$ a known function of $(t, x)$ ) and to the Vlasov-Poisson system (1-3). Then, in $\S 3$, we estimate the mild solutions to the linear Vlasov equation with initial data in $W^{1,1}\left(\mathbb{R}^{2}\right)$, and use these results to construct a contraction mapping on a suitable set, whose fixed point gives a mild solution to the Vlasov-Poisson system. Finally, we extend these results to initial conditions lying in $B V\left(\mathbb{R}^{2}\right)$ in $\S 4$.

\section{Weak and mild solutions}

\subsection{Definition of a weak solution}

We recall the definition of a weak solution to (1-3) by using the spaces of test functions and the functionals introduced by Guo in [11]. We define two spaces of test functions, one for the Vlasov equation and the other for the Poisson equation:

$$
\mathcal{V}=C_{c}^{\infty}\left([0, T) \times \mathbb{R}^{2}\right), \quad \mathcal{M}=C_{c}^{\infty}([0, T) \times \mathbb{R}) .
$$

We define for $\left(E, f, f_{0}\right) \in L_{\text {loc }}^{\infty}\left(U_{T}\right) \times L_{\text {loc }}^{1}\left(V_{T}\right) \times L_{\text {loc }}^{1}\left(\mathbb{R}^{2}\right)$ and $\alpha \in \mathcal{V}$ (still like in [11]) the following functional:

$$
A\left(f, E, f_{0}, \alpha\right)=\int_{\mathbb{R}^{2}} f_{0}(x, v) \alpha(0, x, v) d x d v+\int_{0}^{T} \int_{\mathbb{R}^{2}}\left[\left(Y_{E} \alpha\right) f\right](t, x, v) d x d v d t
$$

We define for $\left(E, f, n_{\mathrm{b}}\right) \in L_{\text {loc }}^{\infty}\left(U_{T}\right) \times L_{\text {loc }}^{1}\left((0, T) \times \mathbb{R}_{\times} ; L^{1}\left(\mathbb{R}_{V}\right)\right) \times L_{\text {loc }}^{\infty}\left(U_{T}\right)$ and $\psi \in \mathcal{M}$ the following functional:

$$
C\left(f, E, n_{\mathrm{b}}, \psi\right)=\int_{0}^{T} \int_{\mathbb{R}}\left\{E(t, x) \partial_{x} \psi(t, x)+\psi(t, x)\left[\int_{\mathbb{R}} f(t, x, v) d v-n_{\mathrm{b}}(t, x)\right]\right\} d x d t
$$

These functionals are well-defined.

A weak solution to the linear Vlasov equation associated to $E \in L_{\text {loc }}^{\infty}\left(U_{T}\right)$ with initial condition $f_{0} \in L_{\text {loc }}^{1}\left(\mathbb{R}^{2}\right)$ is a function $f \in L_{\text {loc }}^{1}\left(V_{T}\right)$ which satisfies:

$$
\forall \alpha \in \mathcal{V}, \quad A\left(f, E, f_{0}, \alpha\right)=0 .
$$

A weak solution to the one-dimensional Vlasov-Poisson system with intial condition $f_{0} \in L_{\text {loc }}^{1}\left(\mathbb{R}^{2}\right)$ and neutralising background density $n_{\mathrm{b}} \in L_{\text {loc }}^{\infty}\left(U_{T}\right)$ is a pair $(E, f) \in L_{\text {loc }}^{\infty}\left(U_{T}\right) \times L_{\text {loc }}^{1}\left((0, T) \times \mathbb{R}_{X} ; L^{1}\left(\mathbb{R}_{V}\right)\right)$ which verifies:

$$
\forall(\alpha, \psi) \in \mathcal{V} \times \mathcal{M}, \quad A\left(f, E, f_{0}, \alpha\right)=0 \quad \text { and } \quad C\left(f, E, n_{\mathrm{b}}, \psi\right)=0 .
$$




\subsection{Characteristic curves associated to $E \in X(T)$}

We recall the following results on the characteristic curves of a transport equation, see for example [7] or [20]. Given $E \in X(T)$ and $(t, x, v) \in \overline{V_{T}}$, we consider the differential system :

$$
\begin{aligned}
& \frac{d X}{d s}(s)=V(s) \\
& \frac{d V}{d s}(s)=E(s, X(s)), \\
& (X(t), V(t))=(X, v)
\end{aligned}
$$

As remarked above, this system admits a unique solution for all $(t, x, v) \in \overline{V_{T}}$, which we denote $\Gamma(s ; t, x, v)=$ $(X(s ; t, x, v), V(s ; t, x, v))$ and is called the characteristic curve passing by $(t, x, v)$.

As $E$ is bounded on $[0, T] \times \mathbb{R}$, every characteristic curve is defined from $s=0$ to $s=T$; moreover, the characteristic curves form a partition of $V_{T}$. Thus for every characteristic $\Gamma(s ; t, x, v)$, we can define an origin on $\Pi_{0}: \Gamma(0 ; t, x, v)=$ $(X(0 ; t, x, v), V(0 ; t, x, v))$.

Let $(t, s) \in[0, T]$. We denote by $\phi_{t, s}$ the characteristic flow of $E$, namely the function:

$$
\begin{aligned}
\phi_{t, s}: \mathbb{R}^{2} & \longrightarrow \mathbb{R}^{2} \\
(x, v) & \longmapsto \Gamma(s ; t, x, v) .
\end{aligned}
$$

$\phi_{t, s}$ transports a point $(t, x, v)$ of the slice $\Pi_{t}$ to a point $\left(s, x^{\prime}, v^{\prime}\right)$ of the slice $\Pi_{s}$ by following the characteristic curve passing by $(t, x, v)$. It is well-known that $\phi_{t, s}$ is a bijection (one-to-one and onto mapping) of $\mathbb{R}^{2}$, which admits bounded partial derivatives and whose Jacobian is identically equal to 1 .

\subsection{Definition of a mild solution}

Let $E \in X(T)$ and $(X, V)$ be the associated characteristic curves. A mild solution to the linear Vlasov equation associated to $E$ with initial condition $f_{0} \in L_{\text {loc }}^{1}\left(\mathbb{R}^{2}\right)$ is a function $f \in L_{\text {loc }}^{1}\left(V_{T}\right)$ which satisfies:

$$
f(t, x, v)=f_{0}(X(0 ; t, x, v), V(0 ; t, x, v)) \text { for a.e. }(t, x, v) \in \overline{V_{T}} \text {. }
$$

We recall the following result (see for example [3]):

Proposition 3 Let $E \in X(T)$ and $f_{0} \in L^{1}\left(\mathbb{R}^{2}\right)$. Then $f \in L^{1}\left(V_{T}\right)$ is a weak solution to the linear Vlasov equation associated to $E$ with initial condition $f_{0}$ if and only if it is a mild solution.

This can be shown by using the characteristic change of variables: $(t, x, v) \mapsto\left(t, x_{0}, v_{0}\right)=\left(t, \phi_{t, 0}(x, v)\right)$, as e.g. in Guo [12]. We deduce the existence and uniqueness of a solution $f \in L^{1}\left(V_{T}\right)$ to the linear Vlasov equation associated to a field $E \in X(T)$ :

Corollary 4 Let $E \in X(T)$ and $f_{0} \in L^{1}\left(\mathbb{R}^{2}\right)$. The linear Vlasov equation associated to $E$ with initial condition $f_{0}$ admits a unique weak solution in $L^{1}\left(V_{T}\right)$ defined as: $\forall(t, x, v) \in V_{T}, f(t, x, v)=f_{0}(X(0 ; t, x, v), V(0 ; t, x, v))$.

Finally, a mild solution to the Vlasov-Poisson system with intial condition $f_{0} \in L^{1}\left(\mathbb{R}^{2}\right)$ and neutralising background density $n_{\mathrm{b}} \in L_{\mathrm{loc}}^{\infty}\left(U_{T}\right)$ is defined as a weak solution $(E, f)$, which belongs to $X(T) \times L^{1}\left(V_{T}\right)$, and such that $f$ coincides a.e. with the mild solution to the linear Vlasov equation associated to $E$ with initial condition $f_{0}$.

\section{Proof of Theorem 1}

\subsection{A priori estimates}

The proof of Theorem 1 relies on the following two theorems whose version for a half space is given by Guo in [12].

Theorem 5 Let $E \in X(T)$ and $p \in[1,+\infty)$. We suppose that $u \in L^{p}\left(V_{T}\right)$ and $Y_{E} u \in L^{p}\left(V_{T}\right)$. Then:

1. There exists $u_{0} \in L_{\text {loc }}^{1}\left(\Pi_{0}\right) \simeq L_{\text {loc }}^{1}\left(\mathbb{R}^{2}\right)$, called the trace of $u$ on $\Pi_{0}$, such that $\forall \alpha \in C_{c}^{\infty}\left(\left[0, T\left[\times \mathbb{R}^{2}\right)\right.\right.$,

$$
\int_{V_{T}}\left(Y_{E} u \alpha+u Y_{E} \alpha\right)(t, x, v) d x d v d t=-\int_{\mathbb{R}^{2}} u_{0}(x, v) \alpha(0, x, v) d x d v
$$

2. If $u_{0} \in L^{p}\left(\mathbb{R}^{2}\right)$, then $\forall s \in[0, T], u(s) \in L^{p}\left(\mathbb{R}^{2}\right)$ and

$$
\int_{\mathbb{R}^{2}}|u(s)|^{p} d x d v=\int_{\mathbb{R}^{2}}\left|u_{0}\right|^{p} d x d v+p \int_{0}^{s} \int_{\mathbb{R}^{2}}\left(\operatorname{sgn} u|u|^{p-1} Y_{E} u\right)(\tau) d x d v d \tau
$$


Theorem 6 Let $E \in X(T)$, and $\phi_{t, s}$ be its characteristic flow. We suppose that $u \in L^{1}\left(V_{T}\right)$ and $Y_{E} u \in L^{1}\left(V_{T}\right)$. Let $u_{0}$ be the trace of $u$ on $\Pi_{0}$ defined in Theorem 5. If $K$ is a measurable set of $\mathbb{R}^{2}$ with non-vanishing Lebesgue measure, then:

$$
\int_{\phi_{0, s}(K)}|u(s)| d x d v=\int_{K}\left|u_{0}\right| d x d v+\int_{0}^{s} \int_{\phi_{0, \tau}(K)}\left(\operatorname{sgn} u Y_{E} u\right)(\tau) d x d v d \tau
$$

The proofs rely on the characteristic change of variables and are entirely similar to those of [12].

With these results, we can prove the fundamental estimate on the solutions to the linear Vlasov equation. We introduce the semi-norm $|\cdot|_{W^{1,1}}$ defined by

$$
\forall f \in W^{1,1}\left(\mathbb{R}^{2}\right), \quad|f|_{W^{1,1}}=\left\|\partial_{x} f\right\|_{1}+\left\|\partial_{V} f\right\|_{1}
$$

Theorem 7 Let $E \in X(T)$ and $f_{0} \in W^{1,1}\left(\mathbb{R}^{2}\right)$. Let $f$ be the unique mild solution in $L^{1}\left(V_{T}\right)$ of the linear Vlasov equation associated to $E$ with initial condition $f_{0}$. Then $\forall s \in[0, T], f(s) \in W^{1,1}\left(\mathbb{R}^{2}\right)$ and

$$
\begin{aligned}
& \|f(s)\|_{L^{1}\left(\mathbb{R}^{2}\right)}=\left\|f_{0}\right\|_{L^{1}\left(\mathbb{R}^{2}\right)} ; \\
& |f(s)|_{W^{1,1}\left(\mathbb{R}^{2}\right)} \leq\left|f_{0}\right|_{W^{1,1}\left(\mathbb{R}^{2}\right)} \exp (C(E) s) .
\end{aligned}
$$

Thus, integrating from 0 to $T$ :

$$
\int_{0}^{T}|f(\tau)|_{W^{1,1}\left(\mathbb{R}^{2}\right)} d \tau \leq\left|f_{0}\right|_{W^{1,1}\left(\mathbb{R}^{2}\right)} \frac{\exp (C(E) T)-1}{C(E)} .
$$

Proof. Equation (15) is an immediate consequence of point 2 of Theorem 5 (with $p=1$ ), or of Theorem 6 (with $K=\mathbb{R}^{2}$ ), given that $Y_{E} f=0$.

We now establish the estimate (16) on derivatives. The set of the indefinitely differentiable functions with compact support on $\mathbb{R}^{2}$ is dense in $W^{1,1}\left(\Pi_{0}\right)\left[1\right.$, p. 54]. Thus there exists a sequence $\left(f_{0}^{n}\right)_{n}$ of elements of $C_{c}^{\infty}\left(\mathbb{R}^{2}\right)$, such that $\left\|f_{0}^{n}-f_{0}\right\|_{W^{1,1}\left(\mathbb{R}^{2}\right)} \longrightarrow 0$ when $n \longrightarrow+\infty$.

Similarly, we regularise $E \in L^{\infty}\left(0, T ;, W^{1, \infty}(\mathbb{R})\right)$ in the following way. We define for all $t \in[0, T], E_{n}(t,)=.E(t,). * \rho_{n}$, where $\left(\rho_{n}\right) \in C_{C}^{\infty}\left(\mathbb{R}_{X}\right)$ is a mollifying sequence. The sequence $\left(E_{n}\right)_{n}$ satisfies: $E_{n} \in L^{\infty}\left(0, T ; W^{1, \infty} \cap C^{1}\left(\mathbb{R}_{X}\right)\right) ;\left\|E_{n}\right\|_{L^{\infty}\left(U_{T}\right)} \leq\|E\|_{L^{\infty}\left(U_{T}\right)}$, $\left\|\partial_{x} E_{n}\right\|_{L^{\infty}\left(U_{T}\right)} \leq\left\|\partial_{x} E\right\|_{L^{\infty}\left(U_{T}\right)}$, and $\left\|E-E_{n}\right\|_{L^{\infty}\left(U_{T}\right)} \longrightarrow 0$ when $n \longrightarrow+\infty$. We denote by $\left(X^{n}, V^{n}\right)$ and $\phi_{t, s}^{n}$ the characteristic curves and flow associated to $E_{n}$.

Let $f_{n}$ be the solution to the linear problem associated to $E_{n}$ with initial condition $f_{0}^{n}$; we recall that this solution is given for a.e. $(t, x, v) \in \overline{V_{T}}$ by $f_{n}(t, x, v)=f_{0}^{n}\left(X^{n}(0 ; t, x, v), V^{n}(0 ; t, x, v)\right)$. As $f_{0}^{n}$ is compactly supported, so is $f_{n}$ : its support is contained in the image of the compact $[0, T] \times \operatorname{Supp} f_{0}^{n}$ by the continuous mapping $\left(s, x_{0}, v_{0}\right) \mapsto\left(s, X\left(s ; 0, x_{0}, v_{0}\right), V\left(s ; 0, x_{0}, v_{0}\right)\right)$. Moreover, the characteristics associated to $E_{n}$ are Lipschitz-continuous in all their variables $(s, t, x, v)$, therefore $f_{n} \in W^{1, \infty}\left(V_{T}\right)$.

All together, we have $\partial_{x} f_{n}$ and $\partial_{v} f_{n} \in L_{c}^{\infty}\left(V_{T}\right)$, thus $\partial_{x} f_{n}$ and $\partial_{v} f_{n}$ lie in $L^{1}\left(V_{T}\right)$. Moreover $Y_{E_{n}} \partial_{x} f_{n}=-\partial_{x} E_{n} \partial_{v} f_{n}$ in $\mathcal{D}^{\prime}\left(V_{T}\right)$, thus $Y_{E_{n}} \partial_{x} f_{n}$ lies in $L^{1}\left(V_{T}\right)$. By an integration by parts, it can be shown that the trace of $\partial_{x} f_{n}$ on $\Pi_{0}$ is $\partial_{x} f_{0}^{n}$. If $K$ is a measurable subset of $\mathbb{R}^{2}$ of non-vanishing Lebesgue measure, we get by Theorem 6 :

$$
\int_{\phi_{0, s}^{n}(K)}\left|\partial_{x} f_{n}(s)\right|=\int_{K}\left|\partial_{x} f_{0}^{n}\right|-\int_{0}^{s} \int_{\phi_{0, \tau}^{n}(K)}\left(\operatorname{sgn}\left(\partial_{x} f_{n}\right) \partial_{x} E_{n} \partial_{v} f_{n}\right)(\tau) d \tau ;
$$

for the sake of brevity we have omitted the kinetic integration element $d x d v$. Thus:

$$
\begin{gathered}
\int_{\phi_{0, s}^{n}(K)}\left|\partial_{x} f_{n}(s)\right| \leq \int_{K}\left|\partial_{x} f_{0}^{n}\right|+\left\|\partial_{x} E_{n}\right\|_{L^{\infty}([0, s] \times \mathbb{R})} \int_{0}^{s} \int_{\phi_{0, \tau}^{n}(K)}\left|\partial_{V} f_{n}(\tau)\right| d \tau \\
\int_{\phi_{0, s}^{n}(K)}\left|\partial_{x} f_{n}(s)\right| \leq \int_{K}\left|\partial_{x} f_{0}^{n}\right|+\left\|\partial_{x} E\right\|_{L^{\infty}\left(U_{T}\right)} \int_{0}^{s} \int_{\phi_{0, \tau}^{n}(K)}\left|\partial_{V} f_{n}(\tau)\right| d \tau
\end{gathered}
$$

In the same way, we have $\partial_{V} f_{n} \in L_{1}\left(V_{T}\right)$ and $Y_{E_{n}} \partial_{V} f_{n}=-\partial_{x} f_{n} \in \mathcal{D}^{\prime}\left(V_{T}\right)$, thus $Y_{E_{n}} \partial_{V} f_{n}$ lies in $L^{1}\left(V_{T}\right)$; and one shows that the trace of $\partial_{v} f_{n}$ on $\Pi_{0}$ is $\partial_{v} f_{0}^{n}$. Reasoning as above, we obtain:

$$
\int_{\phi_{0, s}^{n}(K)}\left|\partial_{V} f_{n}(s)\right| \leq \int_{K}\left|\partial_{V} f_{0}^{n}\right|+\int_{0}^{s} \int_{\phi_{0, \tau}^{n}(K)}\left|\partial_{x} f_{n}(\tau)\right| d \tau
$$

We add (18) and (19):

$$
\begin{aligned}
& \int_{\phi_{0, s}^{n}(K)}\left\{\left|\partial_{x} f_{n}(s)\right|+\left|\partial_{v} f_{n}(s)\right|\right\} \leq \int_{K}\left\{\left|\partial_{v} f_{0}^{n}\right|+\left|\partial_{x} f_{0}^{n}\right|\right\} \\
& \quad+\max \left(\left\|\partial_{x} E\right\|_{L^{\infty}\left(U_{T}\right)}, 1\right) \int_{0}^{s} \int_{\phi_{0, \tau}^{n}(K)}\left\{\left|\partial_{x} f_{n}(\tau)\right|+\left|\partial_{v} f_{n}(\tau)\right|\right\} d \tau .
\end{aligned}
$$


Then we utilize the Grönwall lemma, and we get:

$$
\int_{\phi_{0, s}^{n}(K)}\left\{\left|\partial_{x} f_{n}(s)\right|+\left|\partial_{v} f_{n}(s)\right|\right\} \leq \exp (C(E) s) \int_{K}\left\{\left|\partial_{V} f_{0}^{n}\right|+\left|\partial_{x} f_{0}^{n}\right|\right\}
$$

Therefore:

$$
\int_{0}^{T} \int_{\phi_{0, s}^{n}(K)}\left|\nabla f_{n}(s)\right| d s \leq \frac{\exp (C(E) T)-1}{C(E)} \int_{K}\left|\nabla f_{0}^{n}\right|
$$

Now we utilize the Dunford-Pettis weak compactness criterion in $L^{1}$, that can be found for example in $[6$, p. 76$]$ or $[4, p .167]$ :

Theorem 8 (Dunford-Pettis) Let $\left(f_{n}\right)_{n}$ be a bounded sequence of $L^{1}(\Omega)$. The sequence is weakly compact if and only if $\left\{f_{n}\right\}_{n \in \mathbb{N}}$ is equiintegrable, that is to say:

$$
\begin{aligned}
& \forall \epsilon>0, \exists K_{\epsilon} \text { compact } \subset \Omega \text { s.t. } \sup _{n} \int_{\Omega \backslash K_{\epsilon}}\left|f_{n}\right| d \Omega<\epsilon, \quad \text { and: } \\
& \forall \epsilon>0, \exists \eta>0, \forall \mathcal{A} \subset \Omega \text { measurable, meas }(\mathcal{A})<\eta \Longrightarrow \sup _{n} \int_{\mathcal{A}}\left|f_{n}\right| d \Omega<\epsilon .
\end{aligned}
$$

Let $\epsilon>0$. The sequences $\left(\partial_{x} f_{0}^{n}\right)_{n}$ and $\left(\partial_{v} f_{0}^{n}\right)_{n}$ converge in $L^{1}\left(\mathbb{R}^{2}\right)$, thus are weakly compact in $L^{1}\left(\mathbb{R}^{2}\right)$. By the Dunford-Pettis criterion, these sequences are equiintegrable. Thus, there exists a compact $K_{\epsilon}^{0}$ of $\mathbb{R}^{2}$, and $\eta>0$ such that:

$$
\begin{aligned}
& \sup _{n} \int_{\mathbb{R}^{2} \backslash K_{\epsilon}^{0}}\left\{\left|\partial_{x} f_{0}^{n}\right|+\left|\partial_{V} f_{0}^{n}\right|\right\}<\mathrm{e}^{-C(E) T} \epsilon, \quad \text { and: } \\
& \forall \mathcal{A} \subset \mathbb{R}^{2} \text { measurable, } \operatorname{meas}(\mathcal{A})<\eta \Longrightarrow \sup _{n} \int_{\mathcal{A}}\left\{\left|\partial_{x} f_{0}^{n}\right|+\left|\partial_{V} f_{0}^{n}\right|\right\}<\mathrm{e}^{-C(E) T} \epsilon .
\end{aligned}
$$

Let $\mathcal{A}$ be a subset of $\mathbb{R}^{2}$ such that meas $(\mathcal{A}) \leq \eta$. We have for all $n \in \mathbb{N}$, $\operatorname{meas}\left(\phi_{s, 0}^{n}(\mathcal{A})\right)=\operatorname{meas}(\mathcal{A}) \leq \eta$, and we can apply the inequality (20) to get

$$
\sup _{n} \int_{\mathcal{A}}\left|\nabla f_{n}(s)\right| \leq \epsilon .
$$

Thus we see that the sequences $\left(\partial_{x} f_{n}(s)\right)_{n}$ and $\left(\partial_{v} f_{n}(s)\right)_{n}$ verify the second part of the Dunford-Pettis criterion. For the first part of this criterion, we construct a compact $K_{\epsilon}$ such that all the $\phi_{0, s}^{n}\left(K_{\epsilon}^{0}\right) \subset K_{\epsilon}$. Let $\left(X_{L}(s ; t, x, v), V_{L}(s ; t, x, v)\right)$ and $\phi_{t, s}^{L}$ be the characteristic curves and flow associated to free transportation $(E=0)$. Of course, we have : $V_{L}\left(t ; 0, x_{0}, v_{0}\right)=v_{0}$ and $X_{L}\left(t ; 0, x_{0}, v_{0}\right)=x_{0}+v_{0} t$. We denote $L_{\epsilon}=\phi_{0, s}^{L}\left(K_{\epsilon}^{0}\right)$; this set is a compact as the continuous image of a compact. Then, using the estimate on the divergence of characteristics from [7, Lemma 1] or [5, Lemma 4.8], we obtain:

$$
\begin{aligned}
\forall t \in[0, T], \quad & \left|V^{n}\left(t ; 0, x_{0}, v_{0}\right)-V_{L}\left(t ; 0, x_{0}, v_{0}\right)\right| \leq t\left\|E_{n}\right\|_{L^{\infty}\left(U_{t}\right)} \\
& \left|X^{n}\left(t ; 0, x_{0}, v_{0}\right)-X_{L}\left(t ; 0, x_{0}, v_{0}\right)\right| \leq t^{2}\left\|E_{n}\right\|_{L^{\infty}\left(U_{t}\right)} .
\end{aligned}
$$

Thus we can take for $K_{\epsilon}$ the compact:

$$
\begin{aligned}
K_{\epsilon}=\left\{(x, v) \in \mathbb{R}^{2}: \exists\left(x_{1}, v_{1}\right) \in L_{\epsilon},\left|x-x_{1}\right|\right. & \leq T\|E\|_{L^{\infty}\left(U_{T}\right)} \\
\text { and }\left|v-v_{1}\right| & \left.\leq T^{2}\|E\|_{L^{\infty}\left(U_{T}\right)}\right\} .
\end{aligned}
$$

We have: $\forall n \in \mathbb{N}, \phi_{0, s}^{n}\left(K_{\epsilon}^{0}\right) \subset K_{\epsilon}$. Thus,

$$
\begin{aligned}
& \sup _{n \in \mathbb{N}} \int_{\mathbb{R}^{2} \backslash K_{\epsilon}}\left|\nabla f_{n}(s)\right| \leq \sup _{n \in \mathbb{N}} \int_{\mathbb{R}^{2} \backslash \phi_{0, s}^{n}\left(K_{\epsilon}^{0}\right)}\left|\nabla f_{n}(s)\right| \\
& \quad \leq \sup _{n} \int_{\mathbb{R}^{2} \backslash K_{\epsilon}^{0}}\left\{\left|\partial_{x} f_{0}^{n}\right|+\left|\partial_{V} f_{0}^{n}\right|\right\} e^{C(E) T} \leq \epsilon .
\end{aligned}
$$

Therefore, $\left(\partial_{x} f_{n}(s)\right)_{n}$ and $\left(\partial_{v} f_{n}(s)\right)_{n}$ verify the Dunford-Pettis criterion and thus converge weakly (after extracting a subsequence) in $L^{1}\left(\mathbb{R}^{2}\right)$ toward some functions $g$ and $h$ of $L^{1}\left(\mathbb{R}^{2}\right)$.

On the other hand, we have $Y_{E}\left(f_{n}-f\right)=\left(E-E_{n}\right) \partial_{V} f_{n}$, thus $f_{n}-f$ and $Y_{E}\left(f_{n}-f\right)$ are in $L^{1}\left(V_{T}\right)$. Applying point 2 of Theorem 5 and then the bound (21), we find:

$$
\begin{aligned}
\int_{\mathbb{R}^{2}}\left|f(s)-f_{n}(s)\right| & \leq \int_{\mathbb{R}^{2}}\left|f_{0}-f_{0}^{n}\right|+\int_{0}^{s} \int_{\mathbb{R}^{2}}\left|E(\tau)-E_{n}(\tau)\right|\left|\partial_{v} f_{n}(\tau)\right| d \tau \\
& \leq \int_{\mathbb{R}^{2}}\left|f_{0}-f_{0}^{n}\right|+\left\|E-E_{n}\right\|_{L^{\infty}\left(U_{T}\right)} \frac{\exp (C(E) T)-1}{C(E)} \int_{\mathbb{R}^{2}}\left|\nabla f_{0}^{n}\right| .
\end{aligned}
$$


Thus, $f_{n}(s)$ converges toward $f(s)$ in $L^{1}\left(\mathbb{R}^{2}\right)$. As a consequence, $\partial_{x} f_{n}(s)$ and $\partial_{v} f_{n}(s)$ converge toward $\partial_{x} f(s)$ and $\partial_{v} f(s)$ in $\mathcal{D}^{\prime}\left(\mathbb{R}^{2}\right)$; therefore $g=\partial_{x} f(s)$ and $h=\partial_{v} f(s)$, i.e. $\partial_{x} f(s)$ and $\partial_{v} f(s)$ lie in $L^{1}\left(\mathbb{R}^{2}\right)$. In other words, $f(s)$ appears as the weak limit in $W^{1,1}\left(\mathbb{R}^{2}\right)$ of the sequence $\left(f_{n}(s)\right)_{n}$. By passing to the limit in $(20)$, we get:

$$
\int_{\mathbb{R}^{2}}|\nabla f(s)| \leq \liminf _{n \rightarrow+\infty} \int_{\mathbb{R}^{2}}\left|\nabla f_{n}(s)\right| \leq \exp (C(E) s)\left\|\nabla f_{0}\right\|_{L^{1}\left(\mathbb{R}^{2}\right)}
$$

which is (16), and yields (17) by integrating from 0 to $T$.

\subsection{Construction of a contraction mapping}

We now study the non-linear Vlasov-Poisson problem. In the rest of $\S 3$, we choose $f_{0} \in W^{1,1}\left(\mathbb{R}^{2}\right)$ and $n_{\mathrm{b}} \in L_{\text {loc }}^{\infty}\left(\mathbb{R}^{+} ; L^{1}(\mathbb{R}) \cap\right.$ $L^{\infty}(\mathbb{R})$ ), both being non-negative. In order to construct a contraction mapping from a closed subset of a Banach space to itself, we define the following mappings:

- $\rho: L^{1}\left(\mathbb{R}^{2}\right) \rightarrow L^{1}(\mathbb{R})$ maps $g \in L^{1}\left(\mathbb{R}^{2}\right)$ to its spatial density defined as: $\forall x \in \mathbb{R}, \rho[g](x)=\int_{-\infty}^{+\infty} g(x, v) d v$;

- $\phi_{1}: X(T) \rightarrow L^{\infty}\left(0, T ; L^{1}\left(\mathbb{R}^{2}\right)\right)$ maps $E \in X(T)$ to the unique mild solution $f$ to the linear Vlasov equation associated to $E$ and with initial condition $f_{0}$;

- $\phi_{2}: L^{\infty}\left(0, T ; L^{1}\left(\mathbb{R}^{2}\right)\right) \rightarrow L^{\infty}\left(U_{T}\right)$ maps $f \in L^{\infty}\left(0, T ; L^{1}\left(\mathbb{R}^{2}\right)\right)$ to the function $\mathcal{E}$ defined as:

$$
\mathcal{E}(t, x)=\frac{1}{2} \int_{-\infty}^{x}\left(\rho[f(t)](y)-n_{\mathrm{b}}(t, y)\right) d y-\frac{1}{2} \int_{x}^{+\infty}\left(\rho[f(t)](y)-n_{\mathrm{b}}(t, y)\right) d y .
$$

Of course, it satisfies $\partial_{x} \mathcal{E}=\rho[f]-n_{\mathrm{b}}$, thus it is a solution to the Poisson equation (2). This particular choice corresponds to the fundamental solution $G(x)=\frac{1}{2} \operatorname{sgn}(x)=\frac{1}{2} x /|x|$, the counterpart of those appearing in higher dimensions.

The following lemma will be crucial in our proof. It is left as an exercise to the reader.

Lemma 9 For $R \geq 0$, let $B_{R}^{\prime}$ be the closed ball of center 0 and radius $R$ of the Banach space $\left(X(T),\|\cdot\|_{X(T)}\right)$. Then, $B_{R}^{\prime}$ is a closed subset of the Banach space $\left(L^{\infty}\left(U_{T}\right),\|\cdot\|_{\infty}\right)$, hence it is complete for this norm.

Then we state and prove the following property of the mapping $\rho$ :

Lemma 10 Let $g \in W^{1,1}\left(\mathbb{R}^{2}\right)$. Then, $\rho[g] \in L^{\infty}(\mathbb{R})$ and $\|\rho[g]\|_{\infty} \leq\left\|\partial_{x} g\right\|_{1} \leq|g|_{W^{1,1}}$.

Proof. By Fubini's theorem, the mapping $x \mapsto g(x, v)$ is in $W^{1,1}(\mathbb{R})$, for a.e. $v \in \mathbb{R}$, hence it satisfies $\lim _{x} \rightarrow-\infty g(x, v)=0$. We have thus:

$$
\begin{aligned}
& \rho[g](x)=\int_{-\infty}^{+\infty} g(x, v) d v=\int_{-\infty}^{+\infty} \int_{-\infty}^{x} \partial_{x} g(y, v) d y d v \\
& |\rho[g](x)| \leq \int_{-\infty}^{+\infty} \int_{-\infty}^{x}\left|\partial_{x} g(y, v)\right| d y d v \leq\left\|\partial_{x} g\right\|_{L^{1}\left(\mathbb{R}^{2}\right)}
\end{aligned}
$$

hence the result.

3.2.1. Stability and Lipschitz continuity of $\phi_{2} \circ \phi_{1}$ Let $E \in X(T)$. Theorem 7 gives $\phi_{1}(E) \in L(T)$; moreover we have:

$$
\left\|\phi_{1}(E)\right\|_{L^{\infty}\left(0, T ; L^{1}\left(\mathbb{R}^{2}\right)\right)}=\left\|f_{0}\right\|_{1} ; \quad\left\|\nabla \phi_{1}(E)\right\|_{L^{\infty}\left(0, T ; L^{1}\left(\mathbb{R}^{2}\right)\right)} \leq\left|f_{0}\right|_{W^{1,1}} \mathrm{e}^{C(E) T} .
$$

Let $f \in L(T)$. From the definition of $\phi_{2}$, we deduce: $\left|\phi_{2}(f)(t, x)\right| \leq \frac{1}{2}\left\|\rho[f](t)-n_{\mathrm{b}}(t)\right\|_{L^{1}(\mathbb{R})}$, and thus

$$
\left\|\phi_{2}(f)\right\|_{L^{\infty}\left(U_{T}\right)} \leq \frac{1}{2}\left[\|f\|_{L^{\infty}\left(0, T ; L^{1}\left(\mathbb{R}^{2}\right)\right)}+\left\|n_{\mathrm{b}}\right\|_{L^{\infty}\left(0, T ; L^{1}(\mathbb{R})\right)}\right] .
$$

Assume that $f$ is non-negative; thus the same holds for $\rho[f(t)]$, for a.e. $t$. The equation $\partial_{x} \phi_{2}(f)(t, x)=\rho[f(t)](x)-n_{\mathrm{b}}(t, x)$ gives $\left|\partial_{x} \phi_{2}(f)(t, x)\right| \leq \max \left(\rho[f(t)](x), n_{\mathrm{b}}(t, x)\right)$. By Lemma 10, we infer:

$$
\left\|\partial_{x} \phi_{2}(f)\right\|_{L^{\infty}([0, T] \times \mathbb{R})} \leq \max \left(\left\|\partial_{x} f\right\|_{L^{\infty}\left([0, T], L^{1}\left(\mathbb{R}^{2}\right)\right)},\left\|n_{\mathrm{b}}\right\|_{L^{\infty}([0, T] \times \mathbb{R})}\right) .
$$

Together with (28), we obtain the following bound

$$
\left\|\phi_{2}(f)\right\|_{X(T)} \leq \max \left\{\frac{1}{2}\left[\|f\|_{L^{\infty}\left(0, T ; L^{1}\left(\mathbb{R}^{2}\right)\right)}+\left\|n_{\mathrm{b}}\right\|_{L^{\infty}\left(0, T ; L^{1}(\mathbb{R})\right)}\right],\left\|n_{\mathrm{b}}\right\|_{L^{\infty}([0, T] \times \mathbb{R})},\left\|\partial_{X} f\right\|_{L^{\infty}\left([0, T], L^{1}\left(\mathbb{R}^{2}\right)\right)}\right\} .
$$

Now, if $f=\phi_{1}(E)$, it is non-negative as soon as the initial data $f_{0}$ is. We combine (29) and (27) and find:

$$
\begin{aligned}
& \forall E \in X(T), \quad\left\|\phi_{2} \circ \phi_{1}(E)\right\|_{X(T)} \leq \max \left\{M_{T}, M_{T}^{\prime},\left|f_{0}\right|_{W^{1,1}\left(\mathbb{R}^{2}\right)} \mathrm{e}^{C(E) T}\right\}, \\
& \text { where: } \quad M_{T}:=\frac{1}{2}\left[\left\|f_{0}\right\|_{L^{1}\left(\mathbb{R}^{2}\right)}+\left\|n_{\mathrm{b}}\right\|_{L^{\infty}\left(0, T ; L^{1}(\mathbb{R})\right)}\right], \quad M_{T}^{\prime}:=\left\|n_{\mathrm{b}}\right\|_{L^{\infty}\left(U_{T}\right)} .
\end{aligned}
$$


Now we show that $\phi_{2} \circ \phi_{1}$ is a Lipschitz-continuous mapping in the norm of $L^{\infty}\left(U_{T}\right)$. Let $E_{1}, E_{2} \in X(T)$; we denote $f_{1}=\phi_{1}\left(E_{1}\right)$ and $f_{2}=\phi_{1}\left(E_{2}\right)$. There holds: $Y_{E_{1}}\left(f_{1}-f_{2}\right)=\left(Y_{E_{2}}-Y_{E_{1}}\right)\left(f_{2}\right)=\left(E_{2}-E_{1}\right) \partial_{V} f_{2}$. Thus, $\left(f_{1}-f_{2}\right) \in L^{1}\left(V_{T}\right)$ and $Y_{E_{1}}\left(f_{1}-f_{2}\right) \in L^{1}\left(V_{T}\right)$; we apply Theorem 5 and find:

$$
\int_{\mathbb{R}^{2}}\left|f_{1}(s)-f_{2}(s)\right| \leq \int_{0}^{s} \int_{\mathbb{R}^{2}}\left|E_{1}(\tau)-E_{2}(\tau)\right|\left|\partial_{v} f_{2}(\tau)\right| d \tau
$$

Thus $\left\|f_{1}-f_{2}\right\|_{L^{\infty}\left(0, T ; L^{1}\left(\mathbb{R}^{2}\right)\right)} \leq\left\|E_{1}-E_{2}\right\|_{L^{\infty}\left(U_{T}\right)}\left\|\partial_{V} f_{2}\right\|_{L^{1}\left(V_{T}\right)}$; applying the bound (17), we obtain:

$$
\begin{aligned}
& \| \phi_{1}\left(E_{1}\right)- \phi_{1}\left(E_{2}\right) \|_{L^{\infty}\left(0, T ; L^{1}\left(\mathbb{R}^{2}\right)\right)} \leq \\
&\left\|E_{1}-E_{2}\right\|_{L^{\infty}\left(U_{T}\right)}\left|f_{0}\right|_{W^{1,1}\left(\mathbb{R}^{2}\right)} \frac{\exp \left(C\left(E_{2}\right) T\right)-1}{C\left(E_{2}\right)} .
\end{aligned}
$$

As for $\phi_{2}$, it is an affine mapping, the associated linear mapping corresponding to the case $n_{\mathrm{b}} \equiv 0$. Therefore, for $f_{1}, f_{2} \in L(T)$, one can apply the bound (28) with $f=f_{1}-f_{2}$ and $n_{\mathrm{b}} \equiv 0$ :

$$
\left\|\phi_{2}\left(f_{1}\right)-\phi_{2}\left(f_{2}\right)\right\|_{L^{\infty}\left(U_{T}\right)} \leq \frac{1}{2}\left\|f_{1}-f_{2}\right\|_{L^{\infty}\left(0, T ; L^{1}\left(\mathbb{R}^{2}\right)\right)} .
$$

Finally we arrive at:

$$
\begin{aligned}
\left\|\phi_{2} \circ \phi_{1}\left(E_{1}\right)-\phi_{2} \circ \phi_{1}\left(E_{2}\right)\right\|_{L^{\infty}\left(U_{T}\right)} \leq \\
\frac{1}{2}\left\|E_{1}-E_{2}\right\|_{L^{\infty}\left(U_{T}\right)}\left|f_{0}\right|_{W^{1,1}\left(\mathbb{R}^{2}\right)} \frac{\exp \left(C\left(E_{2}\right) T\right)-1}{C\left(E_{2}\right)} .
\end{aligned}
$$

3.2.2. Local existence and uniqueness We now give conditions on the parameters $R$ and $T$ in order to have: (i) the closed ball $B_{R}^{\prime}$ stable by $\phi_{2} \circ \phi_{1}$, and (ii) $\phi_{2} \circ \phi_{1}$ a contraction mapping on $B_{R}^{\prime}$. In order to bound the various norms of $n_{\mathrm{b}}$, we settle on an arbitrary finite time interval $\left(0, T_{*}\right)$; notice that $M_{T}$ and $M_{T}^{\prime}$ are non-decreasing functions of $T$. The stability estimate (30) implies (i) provided: $\left|f_{0}\right|_{W^{1,1}\left(\mathbb{R}^{2}\right)} \exp (\max (R, 1) T) \leq R$ and $M_{T_{*}}, M_{T_{*}}^{\prime} \leq R$. Thus we choose:

$$
R \geq \max \left(\left|f_{0}\right|_{W^{1,1}\left(\mathbb{R}^{2}\right)}, M_{T_{*}}, M_{T_{*}}^{\prime}\right) \quad \text { and } \quad T \leq \frac{1}{\max (R, 1)} \ln \left(\frac{R}{\left|f_{0}\right|_{W^{1,1}\left(\mathbb{R}^{2}\right)}}\right) .
$$

As for the point (ii), the Lipschitz estimate (33) yields the sufficient condition $R \geq 1$ and $\frac{1}{2}\left|f_{0}\right|_{W^{1,1}\left(\mathbb{R}^{2}\right)}(\exp (R T)-1) / R<1$. We take for example:

$$
R \geq \max \left(1,\left|f_{0}\right|_{W^{1,1}\left(\mathbb{R}^{2}\right)}\right) \quad \text { and } \quad T<\frac{1}{R} \ln \left(1+\frac{2 R}{\left|f_{0}\right|_{W^{1,1}\left(\mathbb{R}^{2}\right)}}\right) .
$$

Considering the two conditions, we obtain that given

$$
R \geq \max \left(1,\left|f_{0}\right|_{W^{1,1}\left(\mathbb{R}^{2}\right)}, M_{T_{*}}, M_{T_{*}}^{\prime}\right), \quad T \leq \min \left(T_{*}, \frac{1}{R} \ln \left(\frac{R}{\left|f_{0}\right|_{W^{1,1}\left(\mathbb{R}^{2}\right)}}\right)\right)
$$

the mapping $\phi_{2} \circ \phi_{1}$ goes from $B_{R}^{\prime}$ into $B_{R}^{\prime}$ and is a contraction for the norm $\|\cdot\|_{L^{\infty}\left(U_{T}\right)}$. By Lemma $9, B_{R}^{\prime}$ is a complete space for this norm. Utilizing the contraction mapping principle, the mapping $\phi_{2} \circ \phi_{1}$ admits a unique fixed point $E \in B_{R}^{\prime}$. If we denote $f=\phi_{1}(E)$, the pair $(E, f) \in X(T) \times L(T)$ is a mild solution to $(1-3)$.

3.2.3. Estimation of the existence time Assume that $T_{*}$ is large enough; we define $R_{0}=\max \left(1, M_{T_{*}}, M_{T_{*}}^{\prime}\right)$. The function $x \mapsto \ln (a x) / x$ admits a unique maximum at the point $x=\mathrm{e} / a$, and its value is $a / e$. Thus, the greatest value of expression $\frac{1}{R} \ln \left(\frac{R}{\left|f_{0}\right|_{W^{1,1}}}\right)$ is attained at $R=\mathrm{e}\left|f_{0}\right|_{W^{1,1}}$ and equal to $\left(\mathrm{e}\left|f_{0}\right|_{W^{1,1}}\right)^{-1}$.

There are two possibilities. If $\left|f_{0}\right|_{W^{1,1}} \geq R_{0} / \mathrm{e}$, we can take $R=R_{1}:=\mathrm{e}\left|f_{0}\right|_{W^{1,1}}$ and $T=T_{1}:=\left(\mathrm{e}\left|f_{0}\right|_{W^{1,1}}\right)^{-1}$ in $\S 3.2 .2$. The estimate (16) then shows $\left|f\left(T_{1}\right)\right|_{W^{1,1}}=\mathrm{e}\left|f_{0}\right|_{W^{1,1}}$. So, $\$ 3.2 .2$ proves the existence and uniqueness of the solution to the Vlasov-Poisson problem with initial data $f\left(T_{1}\right)$ during the time $T_{2}:=\left(\mathrm{e}\left|f\left(T_{1}\right)\right|_{W_{1,1}}\right)^{-1}=\left(\mathrm{e}^{2}\left|f_{0}\right|_{W^{1,1}}\right)^{-1}$. The local existence and uniqueness result allows one to glue together solutions to $(1-2)$ that coincide at some time $T_{1}$. Thus, the solution generated by the initial data $f_{0}$ exists during $T_{1}+T_{2}$. By induction, we obtain an existence time at least equal to:

$$
\frac{1}{\left|f_{0}\right|_{W^{1,1}}}\left(\frac{1}{\mathrm{e}}+\frac{1}{\mathrm{e}^{2}}+\cdots+\frac{1}{\mathrm{e}^{n}}+\cdots\right)=\frac{1}{(\mathrm{e}-1)\left|f_{0}\right|_{W^{1,1}}}
$$

provided this quantity is still less or equal to $T_{*}$. Now, if $\left|f_{0}\right|_{W^{1,1}} \leq R_{0} / \mathrm{e}$, the existence time given by $\S 3.2 .2$ is maximal for $R=R_{0}$ and equal to is equal to $T_{0}:=\frac{1}{R_{0}} \ln \left(\frac{R_{0}}{\left|f_{0}\right|_{W^{1,1}}}\right)$. Applying (16), we obtain $\left|f\left(T_{0}\right)\right|_{W^{1,1}}=\mathrm{e}^{R_{0} T_{0}}\left|f_{0}\right|_{W^{1,1}}=R_{0}>R_{0} /$ e. Thus we can use the previous argument to show that the solution to the Vlasov-Poisson problem with initial data $f\left(T_{0}\right)$ exists for a time at least equal to $\left((\mathrm{e}-1) R_{0}\right)^{-1}$. Finally, the total existence time is no less than

$$
\frac{1}{R_{0}}\left(\ln \left(\frac{R_{0}}{\left|f_{0}\right|_{W^{1,1}}}\right)+\frac{1}{(\mathrm{e}-1)}\right)
$$

if this is no more than $T_{*}$. 
3.2.4. Blow-up versus globality Consider the maximal solution $(f, E)$ to $(1-3)$ with data $f_{0}$ and $n_{\mathrm{b}}$, i.e. that with the largest existence interval $\left(0, T_{\text {ex }}\right)$. Assume that $T_{\text {ex }}$ is finite. Then, we show that $\lim _{t \rightarrow T_{\text {ex }}}|f(t)|_{W_{1,1}=+\infty}$. Otherwise, there would exist a sequence $\left(t_{k}\right)_{k}$ such that $t_{k} \rightarrow T_{\text {ex }}$ and $\left|f\left(t_{k}\right)\right|_{W^{1,1}} \leq C$. The argument of $\S 3.2 .3$ would imply that the solution can be extended to an interval $\left(t_{k}, t_{k}+\tau\right)$, with $\tau$ bounded away from 0 . Thus, it could be extended past $T_{\text {ex }}$, a contradiction.

Furthermore, at any instant $t$, the existence time given by $\S 3.2 .3$ cannot be greater than $T_{\text {ex }}-t$. For $t$ close enough to $T_{\text {ex }}$, $|f(t)|_{W^{1,1}}$ is large enough, and the existence time is equal to $(e-1)^{-1}|f(t)|_{W^{1,1}}^{-1}$; so we get the estimate:

$$
|f(t)|_{W^{1,1}} \geq \frac{1}{(e-1)\left(T_{e x}-t\right)}
$$

Now, the second bound in (27) shows that $\left\|\partial_{x} E(t)\right\|_{\infty}=\left\|\rho[f(t)]-n_{\mathrm{b}}(t)\right\|_{\infty}$ blows up when $t \rightarrow T_{\text {ex }}$. Quantitatively, this norm is greater than $t^{-1} \ln \left(|f(t)|_{W^{1,1}} /\left|f_{0}\right|_{W^{1,1}}\right)$. As the norm of $n_{\mathrm{b}}$ remains bounded on the finite interval $\left(0, T_{*}\right)$, there holds:

$$
\|\rho[f(t)]\|_{\infty} \geq C\left|\ln \left(T_{\text {ex }}-t\right)\right|
$$

for some constant $C$. Conversely, any maximal solution such that $|f(t)|_{W^{1,1}}$ or $\|\rho[f(t)]\|_{\infty}$ remain bounded as $t \rightarrow T_{\text {ex }}$ is global, i.e., $T_{\text {ex }}=+\infty$.

\section{Proof of Theorem 2}

\subsection{Preliminary results}

Here we collect some well-known results on the functions of $W^{1,1}\left(\mathbb{R}^{2}\right)$ and $B V\left(\mathbb{R}^{2}\right)$. The following proposition can be found, for example, in [10, pp. 3-4]:

Proposition $11 W^{1,1}\left(\mathbb{R}^{2}\right) \subset B V\left(\mathbb{R}^{2}\right)$ and $\forall f \in W^{1,1}\left(\mathbb{R}^{2}\right),|f|_{W^{1,1}}=T V[f]$

The following two theorems are taken from [10], p. 7 and p. 14:

Theorem 12 Let $f \in L^{1}\left(\mathbb{R}^{2}\right)$ and $\left(f_{n}\right)_{n}$ be a sequence in $B V\left(\mathbb{R}^{2}\right)$ which converges to $f$ in $L^{1}\left(\mathbb{R}^{2}\right)$. Then:

$$
T V[f] \leq \liminf _{n \longrightarrow+\infty} T V\left[f_{n}\right]
$$

Theorem 13 Let $f \in B V\left(\mathbb{R}^{2}\right)$. There exists a sequence $\left(f_{n}\right)_{n}$ in $C^{\infty}\left(\mathbb{R}^{2}\right) \cap B V\left(\mathbb{R}^{2}\right)$ such that:

$$
\lim _{n \longrightarrow+\infty}\left\|f_{n}-f\right\|_{L^{1}\left(\mathbb{R}^{2}\right)}=0 \quad \text { and } \quad \lim _{n \longrightarrow+\infty} T V\left[f_{n}\right]=T V[f]
$$

\subsection{A priori estimates}

Theorem 14 Let $f \in L^{1}\left(V_{T}\right)$ be the unique mild solution to the linear Vlasov equation associated to $E \in X(T)$ with initial condition $f_{0} \in B V\left(\mathbb{R}^{2}\right)$. Then, $\forall s \in[0, T], f(s) \in B V\left(\mathbb{R}^{2}\right)$ and

$$
T V[f(s)] \leq T V\left[f_{0}\right] \exp (C(E) s)
$$

Thus, integrating from 0 to $T$ :

$$
\int_{0}^{T} T V[f(\tau)] d \tau \leq T V\left[f_{0}\right] \frac{\exp (C(E) T)-1}{C(E)}
$$

Remark that the estimate (15) is still valid, as it only uses the $L^{1}$ character of $f_{0}$ and $f$.

Proof. Let $E, f_{0}$, and $f$ be as in the statement of the theorem. Theorem 13 yields the existence of a sequence $\left(f_{0}^{n}\right)_{n}$ in $C^{\infty}\left(\mathbb{R}^{2}\right) \cap B V\left(\mathbb{R}^{2}\right)$ such that:

$$
\lim _{n \longrightarrow+\infty}\left\|f_{0}^{n}-f_{0}\right\|_{L^{1}\left(\mathbb{R}^{2}\right)}=0 \quad \text { and } \quad \lim _{n \longrightarrow+\infty} T V\left[f_{0}^{n}\right]=T V\left[f_{0}\right]
$$

In particular, we have: $\forall n \in \mathbb{N}, f_{0}^{n} \in W^{1,1}\left(\mathbb{R}^{2}\right)$. 
Let $f_{n}$ be the unique mild solution to the linear Vlasov equation associated to $E$ with initial condition $f_{0}^{n}$. Using Theorem 7 and Proposition 11, we get:

$$
\forall s \in[0, T], \quad T V\left[f_{n}(s)\right] \leq T V\left[f_{0}^{n}\right] \exp (C(E) s) .
$$

We have $Y_{E}\left(f_{n}-f\right)=Y_{E}\left(f_{n}\right)-Y_{E}(f)=0$, so we can use Theorem 6 and obtain:

$$
\int_{\mathbb{R}^{2}}\left|f_{n}(s)-f(s)\right|=\int_{\mathbb{R}^{2}}\left|f_{0}^{n}-f_{0}\right|
$$

Therefore, $\lim f_{n}(s)=f(s)$ in $L^{1}\left(\mathbb{R}^{2}\right)$, for almost every $s \in[0, T]$. Applying Theorem 12 then yields:

$$
\begin{aligned}
T V[f(s)] \leq \liminf _{n \longrightarrow+\infty} T V\left[f_{n}(s)\right] & \leq \liminf _{n \longrightarrow+\infty} T V\left[f_{0}^{n}\right] \exp (C(E) s) \\
& =T V\left[f_{0}\right] \exp (C(E) s),
\end{aligned}
$$

which implies $f(s) \in B V\left(\mathbb{R}^{2}\right)$.

\subsection{Construction of a contraction mapping}

We now get down to the non-linear Vlasov-Poisson problem. We define the mappings $\rho, \phi_{1}$ and $\phi_{2}$ as in $\S 3.2$, and we find sufficient conditions for $\phi_{2} \circ \phi_{1}$ to be a contraction mapping from $B_{R}^{\prime}$ to itself.

Let $f_{0} \in B V\left(\mathbb{R}^{2}\right), E \in X(T)$ and $f=\phi_{1}(E)$. By Theorem 14, $f \in L^{b v}(T)$ and

$$
\left\|\phi_{1}(E)\right\|_{L^{\infty}\left(0, T ; L^{1}\left(\mathbb{R}^{2}\right)\right)}=\left\|f_{0}\right\|_{1} ; \quad\left\|\nabla \phi_{1}(E)\right\|_{L^{\infty}\left(0, T ; L^{1}\left(\mathbb{R}^{2}\right)\right)} \leq T V\left[f_{0}\right] \mathrm{e}^{C(E) T} .
$$

Let us examine the mapping $\phi_{2}$. The bound (28) is still valid. Moreover, if $f$ is non-negative, the inequality $\left|\partial_{x} \phi_{2}(f)(t, x)\right| \leq$ $\max \left(\rho[f(t)](x), n_{\mathrm{b}}(t, x)\right)$ still holds. Combining Lemma 10, Proposition 11 and Theorem 13, one easily proves:

Lemma 15 Let $g \in B V\left(\mathbb{R}^{2}\right)$. Then, $\rho[g] \in L^{\infty}(\mathbb{R})$ and $\|\rho[g]\|_{\infty} \leq T V[g]$.

Thus, one proves the counterpart of the estimate (29):

$$
\left\|\phi_{2}(f)\right\|_{X(T)} \leq \max \left\{\frac{1}{2}\left[\|f\|_{L^{\infty}\left(0, T ; L^{1}\left(\mathbb{R}^{2}\right)\right)}+\left\|n_{\mathrm{b}}\right\|_{L^{\infty}\left(0, T ; L^{1}(\mathbb{R})\right)}\right],\left\|n_{\mathrm{b}}\right\|_{L^{\infty}([0, T] \times \mathbb{R})}, \sup _{t \in[0, T]} T V[f(t)]\right\} .
$$

Together with the estimate on $\phi_{1}$, we obtain:

$$
\forall E \in X(T), \quad\left\|\phi_{2} \circ \phi_{1}(E)\right\|_{X(T)} \leq \max \left(M_{T}, M_{T}^{\prime}, T V\left[f_{0}\right] \mathrm{e}^{C(E) T}\right) .
$$

Now we establish that the mapping $\phi_{2} \circ \phi_{1}$ is Lipschitz continuous in the norm of $L^{\infty}([0, T] \times \mathbb{R})$. Let $E_{1}$, $E_{2} \in X(T)$; we denote $f_{1}=\phi_{1}\left(E_{1}\right)$ and $f_{2}=\phi_{1}\left(E_{2}\right)$. Moreover, as we did in the proof of Theorem 14 , we approximate $f_{0}$ by a sequence $\left(f_{0}^{n}\right)_{n}$ whose terms lie in $W^{1,1}\left(\mathbb{R}^{2}\right)$, and such that

$$
\lim _{n \longrightarrow+\infty}\left\|f_{0}^{n}-f_{0}\right\|_{L^{1}\left(\mathbb{R}^{2}\right)}=0 \quad \text { and } \quad \lim _{n \longrightarrow+\infty} T V\left[f_{0}^{n}\right]=T V\left[f_{0}\right]
$$

The solutions to the linear Vlasov equation with field $E_{1}$ (resp. $\left.E_{2}\right)$ and initial condition $f_{0}^{n}$ will be denoted $f_{1}^{n}\left(\right.$ resp. $\left.f_{2}^{n}\right)$. Applying the $W^{1,1}$ estimate (31) to these functions yields:

$$
\left\|f_{1}^{n}-f_{2}^{n}\right\|_{L^{\infty}\left(0, T ; L^{1}\left(\mathbb{R}^{2}\right)\right)} \leq\left\|E_{1}-E_{2}\right\|_{L^{\infty}\left(U_{T}\right)} T V\left[f_{0}^{n}\right] \frac{\exp \left(C\left(E_{2}\right) T\right)-1}{C\left(E_{2}\right)} .
$$

As seen in the proof of Theorem 14, we have

$$
\left\|f_{i}^{n}(s)-f_{i}(s)\right\|_{L^{1}\left(\mathbb{R}^{2}\right)}=\left\|f_{0}^{n}-f_{0}\right\|_{L^{1}\left(\mathbb{R}^{2}\right)}, \quad \text { for a.e. } s \in[0, T] \text {, and } i=1,2 .
$$

Thus, $f_{i}^{n}$ converges toward $f_{i}$ in $L^{\infty}\left([0, T] ; L^{1}\left(\mathbb{R}^{2}\right)\right)$. Passing to the limit in (34), we obtain:

$$
\left\|f_{1}-f_{2}\right\|_{L^{\infty}\left(0, T ; L^{1}\left(\mathbb{R}^{2}\right)\right)} \leq\left\|E_{1}-E_{2}\right\|_{L^{\infty}\left(U_{T}\right)} T V\left[f_{0}\right] \frac{\exp \left(C\left(E_{2}\right) T\right)-1}{C\left(E_{2}\right)} .
$$

The $L^{1}$ estimate (32) still holds, which finally implies:

$$
\begin{aligned}
\left\|\phi_{2} \circ \phi_{1}\left(E_{1}\right)-\phi_{2} \circ \phi_{1}\left(E_{2}\right)\right\|_{L^{\infty}\left(U_{T}\right)} \leq \\
\frac{1}{2}\left\|E_{1}-E_{2}\right\|_{L^{\infty}\left(U_{T}\right)} T V\left[f_{0}\right] \frac{\exp \left(C\left(E_{2}\right) T\right)-1}{C\left(E_{2}\right)} .
\end{aligned}
$$

Reasoning like in $\S 3.2 .2$, we infer that $\phi_{2} \circ \phi_{1}$ admits a unique fixed point in $B_{R}^{\prime}$ for suitable values of $R$ and $T$ (using the contraction mapping principle of Banach), then we deduce the local existence and uniqueness of a mild solution to (1-3). The existence time is estimated as in $\S 3.2 .3$, and the alternative between blow-up and globality is established as in $\S 3.2 .4$, with obvious adaptations. 


\section{Concluding remarks}

We have established a result of local existence and uniqueness of a mild solution to the one-dimensional Vlasov-Poisson system.

The hypotheses on the data of this problem were improved: the initial data is not assumed to have a compact support, as in [12], or an integrable majorizing function, as in $[5,7,13]$, but only to be of bounded variation. Nothing is assumed, either, about its moments in $v$, unlike most results in higher dimensions $[8,14,15]$. That said, the $B V$ character of the solution implies that the spatial density $\rho[f]$ remains bounded as long as such a solution exists: this property is specific to the dimension one. Therefore, the hypothesis $f_{0} \in B V\left(\mathbb{R}^{2}\right)$ is close to the minimal assumption guaranteeing that $E$ and $\partial_{x} E$ are uniformly bounded, and thus the possibility of the existence of a mild solution in dimension one. Unfortunately, our proof does not extend immediately to higher dimensions.

The drawback is that we were not able to establish global existence. We have seen that this is equivalent to prove that $\rho[f]$ remains bounded on $U_{T}$ for an arbitrary $T$. This is where the more restrictive assumptions made in the literature come in. Interestingly, certain results of uniqueness of weak solutions $[15,16]$ also rest upon the boundedness of the spatial density. The result of this article is, so to say, in the same vein.

Finally, we notice that the arguments presented in this paper can be extended with slight modifications to many-species Vlasov-Poisson systems.

\section{Acknowledgement}

The authors wish to thank the anonymous referee for his interesting remarks, which led to many improvements in the article.

\section{References}

1. Adams RA. Sobolev spaces. Pure and applied mathematics 65, Academic Press, 1975.

2. Batt J. Global symmetric solutions of the initial value problem of stellar dynamics. J. Differential Equations 1977; 25:342-364.

3. Beals R, Protopopescu V. Abstract time-dependent transport equations. J. Math. Anal. Appl. 1987; 121:370-405.

4. Beauzamy B. Introduction to Banach spaces and their geometry. Mathematics studies 68, North-Holland, 1985.

5. Bostan M. Existence and uniqueness of the mild solution for the $1 \mathrm{D}$ Vlasov-Poisson initial-boundary value problem. SIAM J. Math. Anal. 2005; 37:156-188.

6. Brezis H. Analyse fonctionnelle. Mathématiques appliquées pour la maîtrise, Dunod, 1999.

7. Cooper J, Klimas A. Boundary Value Problems for the Vlasov-Maxwell Equation in One Dimension. J. Math. Anal. Appl. 1980; 75:306-329.

8. DiPerna RJ, Lions PL. Global Weak Solutions of the Vlasov-Maxwell System. Comm. Pure. Appl. Math. 1989; XLII:729-757.

9. Filbet F. Contribution à l'analyse et à la simulation numérique de l'équation de Vlasov. Thèse de doctorat, Université Nancy 1, 2001.

10. Giusti E. Minimal surfaces and functions of bounded variation. Monographs in mathematics, Birkhäuser, 1984.

11. Guo Y. Global weak solutions of the Vlasov-Maxwell system with boundary conditions. Commun. Math. Phys. 1993; 174:245-263.

12. Guo Y. Singular Solutions of the Vlasov-Maxwell System on a Half Line. Arch. Rational Mech. Anal. 1995; 131:241-304.

13. Horst, E. On the classical solutions of the initial value problem for the unmodified nonlinear Vlasov Equations. I: General theory. Math. Methods Appl. Sci. 1981; 3:229-248. II: Special cases. Math. Methods Appl. Sci. 1982; 4:19-32.

14. Horst E, Hunze R. Weak Solutions of the Initial Value Problem for the Unmodified Non-linear Vlasov Equation. Math. Meth. Appl. Sci. 1984; 6:262-279.

15. Lions PL, Perthame B. Propagation of moments and regularity for the 3-dimensional Vlasov-Poisson system. Invent. math. 1991; 105:415-430.

16. Loeper G. Uniqueness of the solution to the Vlasov-Poisson system with bounded density. J. Math. Pures Appl. 2006; 86:68-79.

17. Pfaffelmoser K. Global classical solutions to the Vlasov-Poisson system in three dimensions for general initial data. J. Diff. Eq. 1992; 95:281-303.

18. Robert R. Unicité de la solution faible à support compact de l'équation de Vlasov-Poisson, C. R. Acad. Sci. Paris Sér. I Math. 1997; 324: 873-877.

19. Schaeffer J. Global existence of smooth solutions to the Vlasov-Poisson system in three dimensions. Comm. P.D.E. 1991; 16:1313-1335.

20. Ukai S, Okabe T. On classical solutions in the large in time of two-dimensional Vlasov's equation. Osaka J. Math. 1978; 15:245-261.

21. Zhidkov P. On global solutions for the Vlasov-Poisson system. Electronic Journal of Differential Equations 2004:1-11. 